\title{
Correlation between Morphology, Water Uptake, and Proton Conductivity in Radiation- Grafted Proton-Exchange Membranes
}

\author{
Sandor Balog, ${ }^{*}$ Urs Gasser, Kell Mortensen, Lorenz Gubler, \\ Guenther G. Scherer, Hicham Ben youcef
}

An SANS investigation of hydrated proton exchange membranes is presented. Our membranes were synthesized by radiation-induced grafting of ETFE with styrene in the presence of a crosslinker, followed by sulfonation of the styrene. The contrast variation method was used to understand the relationship between morphology, water uptake, and proton conductivity. The membranes are separated into two phases. The amorphous phase hosts the water and swells upon hydration, swelling being inversely proportional to the degree of crosslinking. Hydration and proton conductivity exhibit linear dependence on swelling. Proton conductivity and volumetric fraction of water are related by a power law, indicating a percolated network of finely dispersed aqueous pores in the hydrophilic domains.

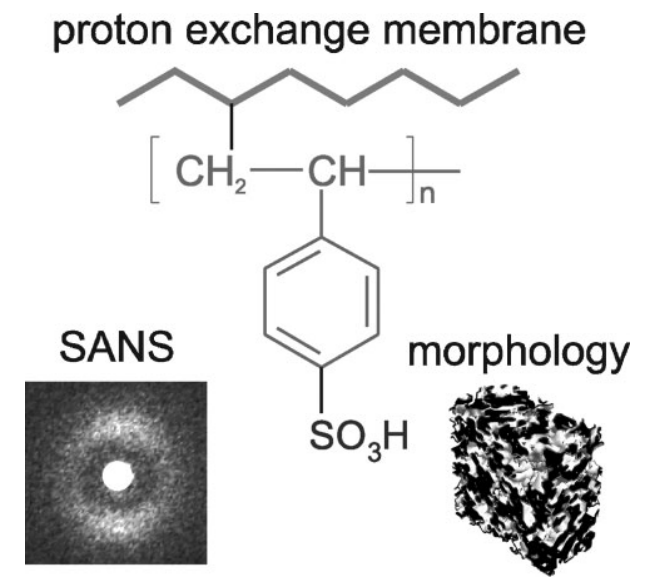

\section{Introduction}

Fuel cells are clean and efficient electrochemical energy conversion reactors. Among the various fuel cell types under consideration, with operating temperatures ranging

S. Balog

Laboratory for Neutron Scattering, ETH Zurich and Paul Scherrer Institut, 5232 Villigen PSI, Switzerland

Fax: +41 56310 2939; E-mail: sandor.balog@psi.ch

U. Gasser

Laboratory for Neutron Scattering, ETH Zurich \& Paul Scherrer Institut, 5232 Villigen, Switzerland, and Adolphe Merkle Institute, University of Fribourg, Route de l'Ancienne Papeterie, P.O. Box 209, 1723 Marly 1, Switzerland

K. Mortensen

Department of Natural Sciences, University of Copenhagen, 1871 Frederiksberg, Denmark

L. Gubler, H. Ben youcef, G. G. Scherer

Electrochemistry Laboratory, Paul Scherrer Institut, 5232 Villigen,

Switzerland up to $1000^{\circ} \mathrm{C}$ for the solid oxide fuel cell (SOFC), the polymer electrolyte fuel cell (PEFC) is particularly attractive for applications with variable load profile and intermittent operation (portable electronics, remote power sources, vehicle propulsion). PEFC typically operate at temperatures between 60 and $100^{\circ} \mathrm{C}$. In a PEFC, the electrochemical reaction of a fuel, typically $\mathrm{H}_{2}$, and $\mathrm{O}_{2}$, which is commonly taken from the ambient air, takes place in two half-cell reactions separated by an electrolyte, which is a polymeric membrane with a thickness of 20 to $200 \mu \mathrm{m}$ and proton conductivity on the order of $0.1 \mathrm{~S} \cdot \mathrm{cm}^{-1} \cdot{ }^{[1]}$ In the PEFC, the electrolyte membrane, allowing transport of protons from anode to cathode, serves at the same time as a separator for electrons and reactant gases. Sulfonic acid groups $\left(-\mathrm{SO}_{3} \mathrm{H}\right)$ with high dissociation constant (low $\mathrm{p} K_{\mathrm{a}}$ ) tethered to the polymer provide protons as charge carriers. In such "ionomers," the dissociation and formation of mobile protons requires the presence of water acting as "proton solvent." The structure of water swollen proton exchange membranes (PEMs), especially Nafion, which is widely used 
in PEFCs, has been subject to numerous investigations. It has been established that Nafion, as well as other wellworking PEMs, have a nano-phase-separated structure, with a "polymer" phase and an "aqueous" phase. ${ }^{[2]}$ The proton transport takes place within water channels lined with sulfonate counter-ions. As a consequence, the mobility of the proton is strongly affected by the water content and pore structure. ${ }^{[3]}$

Although perfluoroalkylsulfonic acid (PFSA) membranes, such as Nafion, are widely used owing their chemical stability and proton properties, the quest for more costeffective partially fluorinated or non-fluorinated materials is ongoing. In addition, the high water uptake ( $\approx 30 \mathrm{wt}$. $\%$ ) of PFSA membranes leads to problems of poor dimensional stability and mechanical degradation in the PEFC. Considering alternative membrane materials, careful design of the ionomer composition, structure, and morphology, e.g., via crosslinking, is expected to improve the structureproperty relationship of these PEMs. It is important to impart the polymer with a property that will promote the spontaneous formation of a biphasic structure. The conceptual decoupling of mechanical support and proton transport properties can be accomplished by several approaches. The formation of polymer blends is a concept that lends itself to combining the properties of dissimilar polymer constituents (Figure 1). Good examples are polyelectrolyte copolymers, comprising units such as styrenesulfonic acid, acrylates, etc., blended into a fluoropolymer matrix. ${ }^{[4]}$ The tunability of phase segregation in blends is governed by the degree of miscibility (FloryHuggins parameter, $\chi$ ), the overall molar mass, and the relative molecular composition.

The synthesis of block copolymers is a versatile strategy, which is pursued by a number of groups, whereby nonsulfonated blocks are combined with sulfonated blocks. ${ }^{[5,6]}$ In a study on polyimide block copolymers, Cornet et al. showed that the structure, determined by small-angle neutron and X-ray scattering, and proton conductivity

\section{polymer blend}
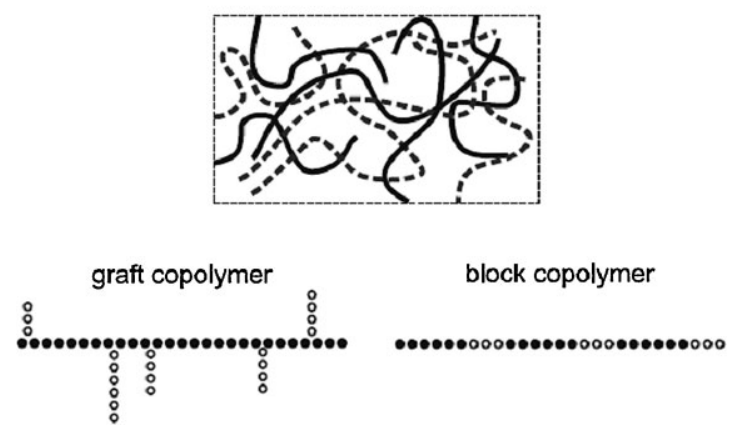

Figure 1. Possible polymer configurations to facilitate the formation of a phase-separated microstructure. The red polymer constituents are units providing proton conductivity. are strongly influenced by the length of the respective sulfonated and non-sulfonated blocks. ${ }^{[7]}$

Graft copolymers represent yet another class of polymers in which two dissimilar polymer constituents may be combined. ${ }^{[8]}$ Graft copolymers are formed by the growth of polymer chains onto reactive sites in the main chain of a base polymer. Generation of the reactive sites may be accomplished by chemical means, in a plasma, or by radiation (UV, $\gamma$-ray, electron, or ion beam). Electrochemical application of ion exchange membranes requires graft copolymerization throughout the film thickness, which necessitates highly penetrating radiation $\left(\mathrm{e}^{-}, \gamma\right)$. Radiation grafting allows the grafting of polar monomers onto non-polar films. ${ }^{[9]}$ For the application in fuel cells, perfluorinated or partially fluorinated films are used for reasons of chemical and thermal stability. Styrene and its derivatives are the most widely used grafting monomers, as the aromatic units are readily sulfonated to introduce ion exchange capacity (IEC). ${ }^{[10]}$

In our laboratory, we have extensively investigated the synthesis and characterization of radiation grafted membranes for application in PEFCs. ${ }^{[1]}$ In this context, crosslinking has been a topic of high interest. Essentially, crosslinking allows the adjustment of the structural density of the grafted component, which has a high influence on the water uptake, conductivity, dimensional stability, and mechanical properties. ${ }^{[12]}$

In a study by Yang and Holdcroft, the structure and properties of well-characterized grafts of poly(styrenesulfonic acid) (PSSA) on a polystyrene (PS) main chain were found to vary systematically with graft side chain length, number density of grafts on the main chain, and total ionic content. ${ }^{[13]}$ Membranes with longer PSSA side chains phase separate to a greater extent for a given ionic content. Membranes with long ionic side chains yet low ionic content possess isolated ionic domains. The increase in the ionic content will lead to coalescence of the ionic domains upon reaching the percolation threshold.

The radiation grafted membranes synthesized in our laboratory have a less well-defined macromolecular configuration. The base polymer is a technical product, and the grafts are obtained by radical polymerization, yielding a non-uniform graft chain length distribution. The addition of a crosslinker further adds to the complexity of the macromolecular structure. Nevertheless, the correlation between morphology, microstructure and fuel cell relevant properties, such as water uptake and proton conductivity, is of profound importance.

In a previous study, we have used small-angle neutron scattering to investigate the domain structure of perfluorinated poly(ethylene-co-propylene) film (FEP) grafted with styrene and crosslinked with divinylbenzene (DVB). ${ }^{[14]}$ Aside from the marked anisotropy caused by the orientation of the base film, we found that the PS is incorporated 
into the amorphous region of the base polymer. Without crosslinker it leads to the formation of large scale structures of several hundred nanometer size. This large scale domain structure formation does not occur when the graft component is crosslinked, thereby retaining the original crystalline/amorphous structure of the FEP base polymer. It has to be made clear, however, that these measurements were done on grafted films only, and not on sulfonated and water swollen membranes, which is expected to introduce further features into the structure of the copolymer.

In this paper, we report on the domain structure and morphology, as observed by small-angle neutron scattering (SANS), of radiation grafted and sulfonated membranes based on poly(ethylene-alt-tetrafluoroethylene) (ETFE) film. Our focus is on the effect of crosslinking on the relationship between the domain structure, water uptake, and proton conductivity.

\section{Experimental Part}

\section{Membrane Synthesis}

The procedure of the membrane synthesis is depicted in Figure 2. ETFE (Tefzel 100LZ) films with $25 \mu \mathrm{m}$ thickness, purchased from DuPont (Circleville, USA), were used as the base polymer. We used electron pre-irradiation method to graft the PS chains. Electron beam irradiation (Leoni Studer AG, Däniken, Switzerland) was performed in air using an accelerating voltage of 2.2 MV and a beam current of 5-20 mA. The applied dose rate was $15.1 \pm 1.1 \mathrm{kGy} \cdot \mathrm{s}^{-1}$. After exposure $(\approx 0.1 \mathrm{~s})$ the films were stored at $-80^{\circ} \mathrm{C}$ until further processing. The reagents used during membrane synthesis were styrene (purum grade; Fluka) and DVB tech. ( $\approx 80 \%$, mixture with isomers 3- and 4-ethylvinylbenzene; Fluka), isopropyl alcohol (analytical grade; Fisher Scientific), dichloromethane (Fluka), and chlorosulfonic acid (Fluka). Grafting reactions were carried out in a stainless steel reactor. The grafting solution consisted of 20 vol.-\% monomer (mixture of styrene and DVB), 65\% isopropyl alcohol, and $15 \%$ water as described previously. ${ }^{[15,16]}$ The degree of grafting, $G_{l}$,

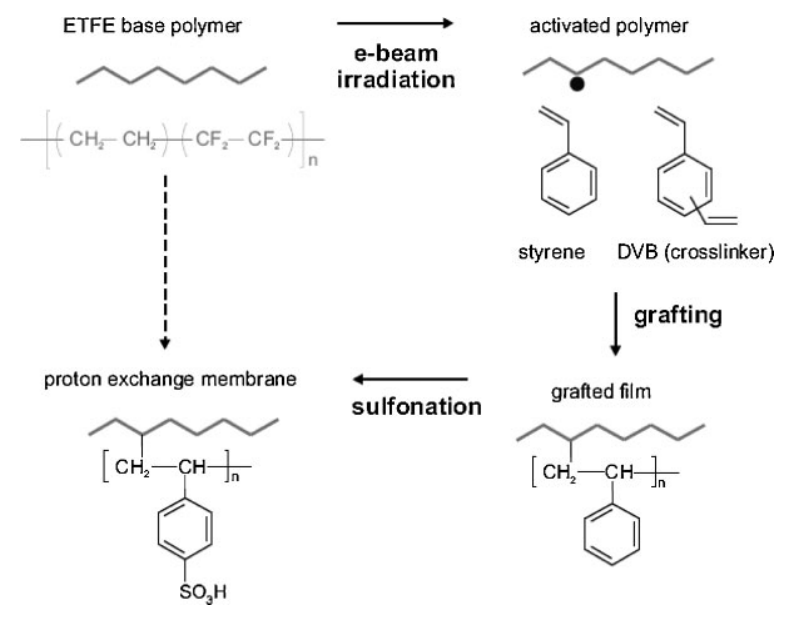

Figure 2. Flow chart of the membrane synthesis. is defined as the relative weight increase of the film

$$
G_{1}=\left(m_{g}-m_{0}\right) / m_{0}
$$

where $m_{0}$ and $m_{g}$ represent the weight of the film before and after grafting, respectively. The DVB level, given as the volume ratio of DVB with respect to the total of monomer (DVB + styrene) in the initial grafting solution, was varied. The grafting reaction was initiated by placing the reactor in a thermostatic bath at $60^{\circ} \mathrm{C}$ and performed for varying reaction times. The grafted films were extracted with toluene, then dried at $80^{\circ} \mathrm{C}$, and finally reweighed in order to determine the graft level.

The membranes were prepared by sulfonating the grafted films with chlorosulfonic acid in dichloromethane, 2 vol. $\%$, at room temperature for 5 hours, followed by hydrolysis in $0.1 \mathrm{M} \mathrm{NaOH}$ solution and re-protonation in $2 \mathrm{M} \mathrm{H}_{2} \mathrm{SO}_{4}$ solution. We swelled the membranes in deionized water at $80^{\circ} \mathrm{C}$ during $5 \mathrm{~h}$.

The IEC, proton conductivity, water uptake, and hydration number of the membranes were characterized using our standard procedures, described elsewhere. ${ }^{[17,18]}$ The thickness of the swollen membrane was measured by using a digital thickness gauge (MT12B Heidenhain, Germany).

\section{Small-Angle Neutron Scattering}

We performed the SANS experiment at the Swiss spallation neutron source (SINQ, Paul Scherrer Institut, Switzerland), using the SANS-II beamline. ${ }^{[19]}$ Each sample (base- and grafted film, the dryand hydrated membrane) is kept at ambient temperature, $23^{\circ} \mathrm{C}$, during the entire experiment. Dry membranes were kept in vacuum and swollen ones were held in closed quartz cells.

For contrast variation we used six different mixtures of deuterated and normal water: (i) $0 / 100$, (ii) $35 / 65$, (iii) $50 / 50$, (iv) $60 / 40$, (v) $75 / 25$, and (vi) $100 / 0$ vol.- $\%$. With these mixtures we covered the range of scattering length densities given for the polymers within the membranes. We recorded scattering at two different configurations of the SANS-II instrument: sample-todetector distance equal to 3 and $6 \mathrm{~m}$ with neutron wavelengths of 0.53 and $1.06 \mathrm{~nm}$, respectively. The collimation length was in both cases equal to the sample-to-detector distance, and the entrance and sample pinholes were 16 and $7 \mathrm{~mm}$, respectively. Raw data were processed according to standard procedures and were calibrated for detector nonlinearity using the incoherent scattering from normal water. We present the scattering spectra as a function of the scattering vector $q=\frac{4 \pi}{\lambda} \sin \frac{\theta}{2}$, where $\theta$ is the scattering angle and $\lambda$ is the wavelength. Sector averaged scattering spectra are created by averaging over an angular range of $\pi / 9$.

\section{Results and Discussion}

\section{Results for Base and Grafted Films}

The scattering spectra of the base film (Figure 3) are azimuthally anisotropic, dominated by a broad correlation peak, and below $0.1 \mathrm{~nm}^{-1}$, we observe an intense upturn in the scattered intensity. The anisotropy of ETFE base film is 


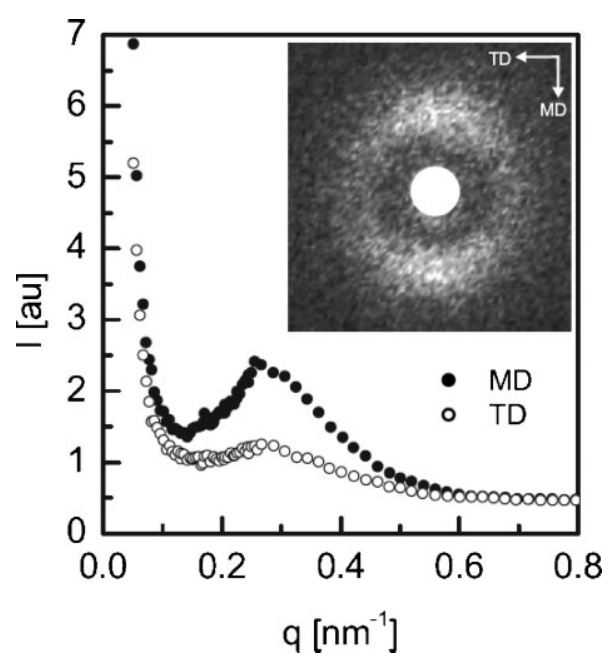

Figure 3. The two-dimensional scattering pattern and the sector averaged curves of the ETFE base film. The full and empty symbols correspond to the machining (MD) and transverse direction (TD).

the result of the extrusion process applied during production. Generally, properties measured in machining direction (MD) are different from the ones in transverse direction (TD). The correlation peak of the TD is shallow compared to that of the MD, but both peaks are centered around $0.28 \mathrm{~nm}^{-1}$ and their widths are practically identical.

The scattering spectra of the grafted films (Figure 4) show basically the same attributes as those of the base film, though the scattered intensity is almost two orders of magnitude higher. The anisotropy is preserved and the scattering is dominated by a correlation peak and a low-angle intensity upturn. Upon grafting, the center of the correlation peak moves to a lower q-position. Both the amplitude and the peak-value tend to reach a saturation upon increasing crosslink level. One may identify an onset of saturation

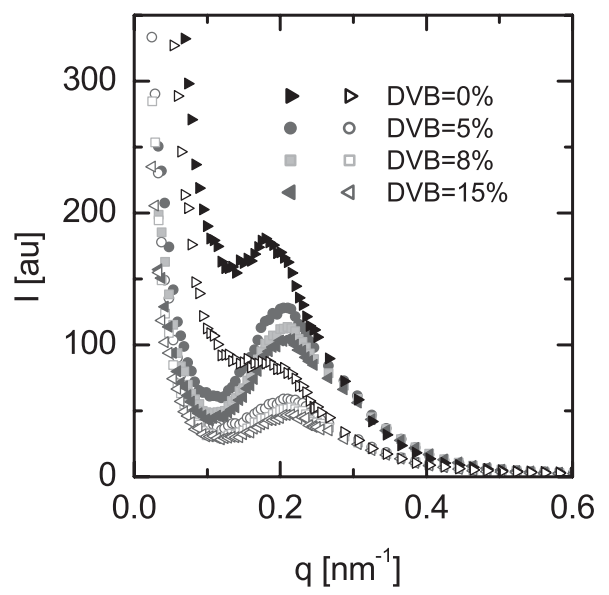

Figure 4. Scattering curves of the grafted films synthesized with various crosslink levels. The graft levels of the films are very similar: $\approx 25 \%$. The full and empty symbols stand for the machining and transverse direction in $q$-space.

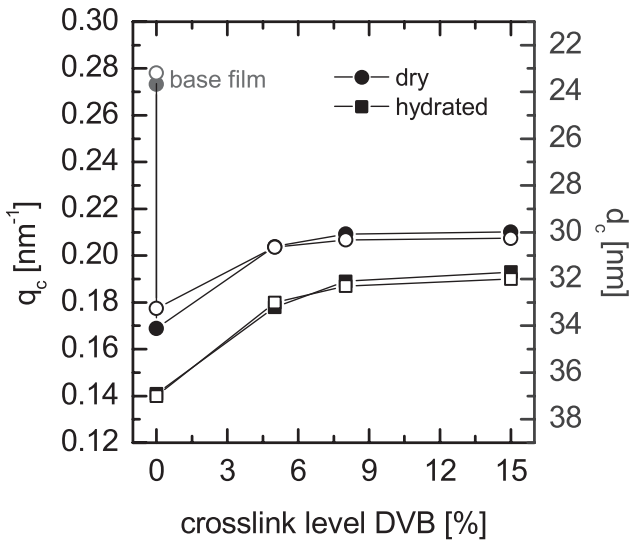

Figure 5. Center of the correlation peaks versus crosslink level for the base film, dry-, and swollen membrane. The full and empty symbols correspond to the machining and transverse direction, respectively. We obtained the center of the correlation peaks by fitting Gaussian functions.

around $8-10 \%$ DVB (Figure 5). The scattering function of the grafted film without crosslinks shows the highest intensity. The correlation peak is rather shallow, seen as a shoulder in the SANS data parallel to the TD.

\section{Results for Dry and Swollen Membranes}

The scattering spectra of the dry membranes are basically identical to those of the grafted films, apart from the overall intensity (Figure 6). We do not observe shifts in the peakvalues or changes in the shape of the peaks.

The scattering spectra of the water swollen membranes are shown in Figure 7. Upon water uptake the position of the correlation peak, $q_{c}$, shifts toward smaller $q$ values (Figure 5). The difference, $\Delta d_{c}$, in the characteristic length, $d_{c} \equiv 2 \pi q_{c}^{-1}$, calculated in the dry and swollen state decreases with increasing crosslink level (Figure 8). The correlation peak of the membrane without crosslinker nearly disappears upon hydration, while for crosslinked membranes, the peaks are well preserved.

Changing the contrast in the membrane synthesized without crosslinker affects only the magnitude of the scattering spectra, thereby indicating a mere biphasic domain structure. On the other hand, for crosslinked membranes the steepness of the low-angle intensity upturn exhibits some dependence on the ratio of the deuterated- and normal water in the mixture, implying a more complex structure in this case.

\section{Discussion of Base and Grafted Films}

The crystallinity of the base film is $34-35 \% .{ }^{[18,20]}$ The presence of a correlation peak, which we identify as the so-called crystalline domain peak, indicates a characteristic 


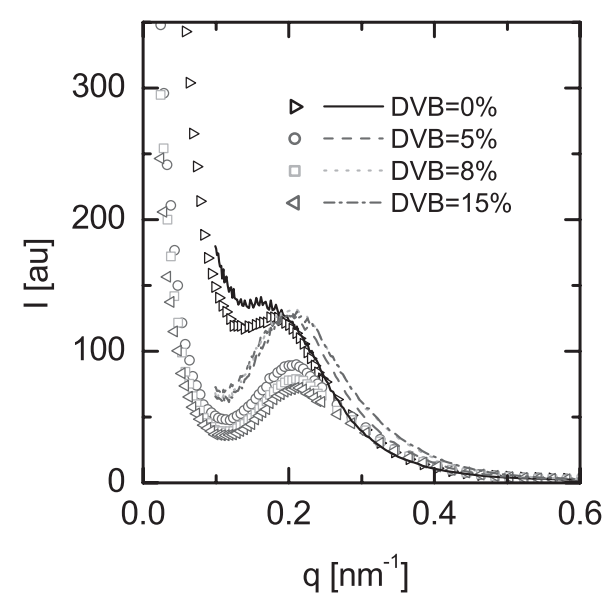

Figure 6. Full azimuthal scattering curves of the grafted films (symbol) and dry membranes (solid line).

length in the morphology, defined by the alternation of the amorphous and crystalline phase. However, the spatial correlation is not sustained but has finite extent, because we do not observe any Bragg-singularity but only one broad correlation peak. Certain soft condensed systems, such as bicontinuous microemulsions ${ }^{[21,22]}$ and bilayer membranes, ${ }^{[23-25]}$ when being in the so-called $L_{3}$ structural phase, show this very particular feature in the scattering spectra. This may indicate that the structure of the amorphous and crystalline phase in the present polymer membranes is bicontinuous and randomly connected.

The increase in the scattered intensity due to grafting reflects the large contrast between PS and ETFE, in relation to the far smaller contrast between amorphous and crystalline ETFE, being the origin of the observed scattering from the base film. However, the scattered intensity decreases with increasing crosslink level. Surprisingly, the SANS data do not indicate any segregation within the amorphous phase, which one could expect as a result of the immiscibility between PS and ETFE.

The crosslinks have an influence on the PS growth, impeding the expansion of the amorphous phase. The incorporation of crosslinks results in interconnected styrene chains, creating a stiff network. Whereas already the smallest amount of crosslink preserves the morphological specifics found in the base film, the growth, and separation of styrene grafted without crosslinks is more spontaneous and less restricted, which may result in sharpening of phase boundaries and developing of larger PS domains. Both effects give rise to an increased scattering intensity. ${ }^{[26]}$ Upon grafting, the peak center also shifts toward smaller $q$ values (Figure 5), which signifies that the characteristic length of the morphology, $d_{c}$, has increased. It is at a maximum without crosslinks, where $d_{c}$ increases from $\approx 22$ to $37 \mathrm{~nm}$. With the increase in crosslink level $d_{c}$ tends to saturate at $\approx 30 \mathrm{~nm}$.

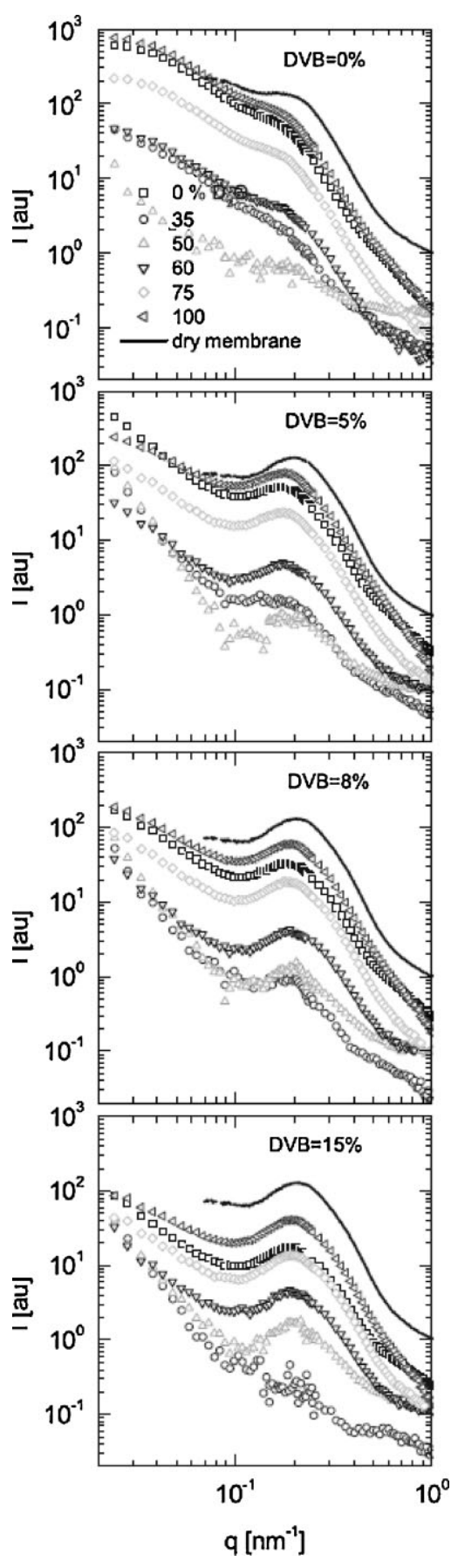

Figure 7. Full azimuthal averaged scattering spectra of the membranes swollen in various mixtures of heavy and light water. For the sake of comparison the dry states of the membranes are also shown.

Prior to this study, we reported that styrene grafts predominantly within the amorphous chains of the backbone matrix. A minor quantity of styrene may graft on the crystalline surfaces but never within. ${ }^{[11]}$ Due to potential bond breaking at the crystalline-amorphous interface, some minor thinning and shortening of the crystallites 


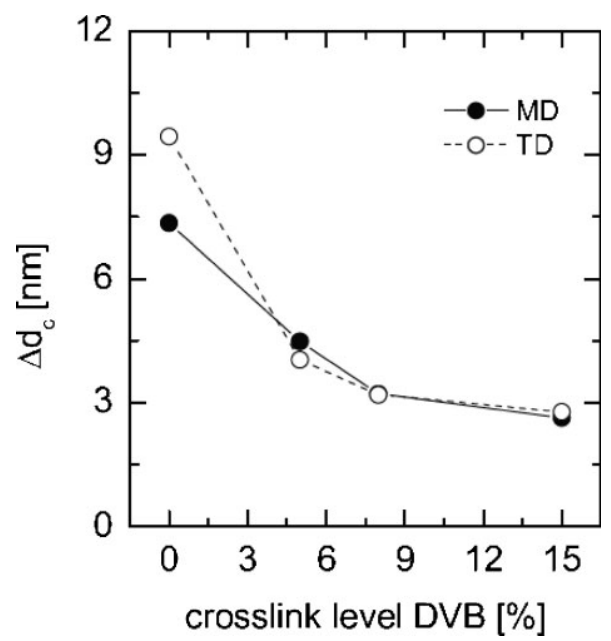

Figure 8. The differences between the characteristic lengths in the dry and the swollen state of the membrane as a function of the crosslink level. The full and empty symbols stand for the machining (MD) and transverse direction (TD).

may be expected upon membrane synthesis. When we incorporate the graft components, the dilution, and the possible disruptions in the crystalline phase have also been observed, though the overall level of crystallinity is more or less preserved, having shown only a minor decrease with increase in crosslink level. ${ }^{[16]}$ Thus, it is the amorphous phase that expands upon accommodating the PS.

We also reported earlier that the crosslinks are more concentrated close to the film surface compared to its interior. ${ }^{[16]}$ Therefore, a crosslinked membrane can be crudely modeled as a "sandwich" of three consecutive layers: external layers with higher level of crosslinks and the inner layer with lower crosslink level. Expansion ratios of the weakly and highly crosslinked domains are substantially different. As SANS spectra result from scattering distributed throughout the entire membrane, this should lead to a distributed scattering function showing up as a spectrum where the correlation peak is markedly broadened. This is not observed. ${ }^{[14]}$ It is most likely due to the fact that already a small amount of crosslink preserves the morphological specifics of the base film.

\section{Discussion of Dry and Swollen Membranes}

We find that the sulfonation of the grafted PS does not affect the SANS spectra (Figure 6), which strongly indicates that the grafted film and the corresponding dry membrane have the same morphology. Upon water uptake, the amorphous domains swell (Figure 5). The relative swelling due to hydration is maximum without crosslinks: the characteristic length, $d_{c}$, increases from $\approx 37$ to $45 \mathrm{~nm}\left(\Delta d_{c} \approx 8 \mathrm{~nm}\right)$, while it appreciably decreases with increasing crosslink level: $d_{\mathrm{c}}$ increases from $\approx 30$ to $33 \mathrm{~nm}\left(\Delta d_{\mathrm{c}} \approx 3 \mathrm{~nm}\right)$, as it is shown in Figure 5 and 8.

The relative change in the volume, $\delta V$, due to water swelling, defined as the difference in volume between the wet and dry state normalized by the dry volume, should be inversely proportional to the relative change in the peak center, $q_{c}$, when assuming isotropic three-dimensional swelling

$$
\delta V=\frac{V^{\text {wet }}-V^{\text {dry }}}{V^{\text {dry }}} \sim\left(\frac{q_{\mathrm{c}}^{\text {dry }}}{q_{\mathrm{c}}^{\text {wet }}}\right)^{3}-1
$$

We have observed earlier that the film upon swelling expands more in thickness direction than in the lateral directions, ${ }^{[16]}$ indicating deviations from the isotropic swelling assumed in Equation (2). Due to lack of structural information perpendicular to the film plane, this is neglected here, yet the volume swelling estimated from our SANS spectra is in very good agreement with the macroscopic results given by measuring the lateral dimensions and thickness of the wet and dry membrane. ${ }^{[16]}$ The agreement is demonstrated by an excellent linear correlation shown in Figure 9, indicating isotropic swelling on the nanometer length scale.

The contrast variation study of the swollen membranes shows that a purely biphasic morphology exists over a length-scale ranging from $\approx 6$ to $60 \mathrm{~nm}$ (Figure 7). The two principal functional phases are the crystalline and the amorphous phase. Although the amorphous phase is composed of chemically different species, the SANS results show that this phase is homogeneous on the aforementioned length-scale. This indicates that in spite of the expected immiscibility between sulfonated PS (SPS) and ETFE there is no molecular segregation within the amorphous phase, i.e., the SPS and adjacent water domains

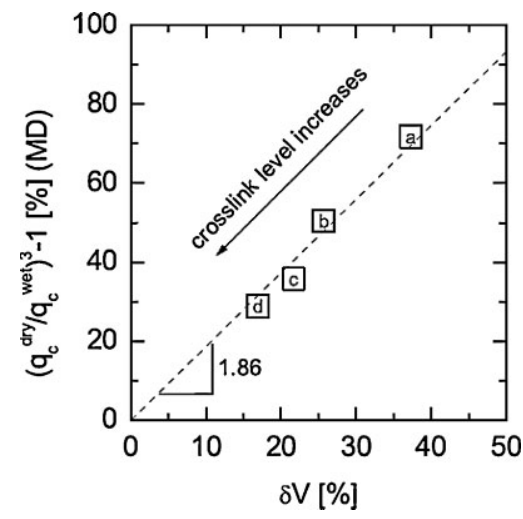

Figure 9. Relative volume change estimated by Equation (2) versus the dimensional change at various crosslink levels. ${ }^{[16]}$ The crosslink level is (a) o, (b) 5, (c) 8, and (d) $15 \%$. The dashed line is the best linear fit. 
must be evenly dispersed within the amorphous ETFE domains.

\section{Discussion of Proton Conductivity and Water Uptake}

The conductivity, $\sigma$, of the membrane depends on several variables: temperature, water content, overall proton concentration, the distribution of local proton concentration inside the water-filled pores, and mobility. ${ }^{[27]}$ The geometry of a pore is decisive. The conductance of a pore depends on the pore length, cross-section, shape, and perimeter. ${ }^{[3]}$ Moreover, these variables are not predefined but evolve as a function of the hydration level. ${ }^{[3,27]}$

Percolation theory relates the conductance of a random composite system to the volumetric fraction of the conducting phase. The conductance shows a power law behavior when the system is near to the percolation threshold, $\sigma \sim\left(v-v_{c}\right)^{t}$, where $v$ is the total volume fraction of the conducting phase and $v_{c}$ is the critical value for the onset of percolation. The universal conductivity exponent for an isotropic porous morphology is $t \approx 2$ in the case of a three-dimensional inert system with rigid pores. ${ }^{[28]} \mathrm{How}^{-}$ ever, an important particularity of polymer electrolyte membranes is the conductivity gradient found along the cross-section of a pore. The gradient is due to the fixed exchange sites, which are $\mathrm{SO}_{3}$-groups in the present study. They influence the concentration and mobility of protons ${ }^{[27,29]}$ and thus, the relationship between $\sigma$ and $v$ is more complicated in a swollen membrane when compared to an inert and rigid system. ${ }^{[30,31]}$ For aqueous PEMs, a greatly simplified empirical law, commonly known as "Bruggeman-like" formula has been asserted, which describes the relationship between conductivity and volume fraction

$$
\sigma \approx \sigma_{\mathrm{r}}+\left(\sigma_{\mathrm{s}}-\sigma_{\mathrm{r}}\right)\left(\frac{v-v_{\mathrm{c}}}{1-v_{\mathrm{c}}}\right)^{t} \text { for } \quad v>v_{\mathrm{c}}
$$

where $\sigma_{\mathrm{r}}$ is the residual conductivity at low water content, which is a few orders of magnitude smaller than the conductivity $\sigma_{\mathrm{s}}$ of the saturated sample. Above but near the threshold $1.3 \leq t \leq 1.7 .^{[32]}$ When $v$ is considerably higher than the value of $v_{c}$, after having passed a transition regime, the critical exponent has been found to be smaller than unity. For a three-dimensional random system, $v_{c}$ has been found to be around 15\%. This value may be larger if the ionic clusters are separated into isolated domains, and may be smaller when segregation is less, so that an extended and continuous network is built up. ${ }^{[32]}$ For example, low threshold percolation values have been found for conductive polymer blends. ${ }^{[33,34]}$

Based on our phenomenological model for water uptake explained below, we are able to determine the volume fractions of the chemical components [ETFE, SPS, and water $(\mathrm{H})]$ in the swollen state of the membrane as a function of the crosslink level. First we describe the mass at each step of the membrane synthesis: ETFE base film, $m_{\mathrm{ETFE}}$; grafted film, $m_{\mathrm{ETFE}}+m_{\mathrm{PS}}+m_{\mathrm{DVB}}$; dry membrane (sulfonated grafted film), $m_{\mathrm{ETFE}}+m_{\mathrm{SPS}}+m_{\mathrm{DVB}}$; and the swollen membrane, $m_{\mathrm{ETFE}}+m_{\mathrm{SPS}}+m_{\mathrm{DVB}}+m_{\mathrm{H}}$, where $m_{i}$ stands for the mass of the incorporated chemical compounds and $m_{\mathrm{SPS}}=m_{\mathrm{PS}}+m_{\mathrm{S}}$. The degree of grafting is defined by Equation (1). Accordingly, $G_{l}=\left(m_{\mathrm{PS}}+m_{\mathrm{DVB}}\right) / m_{\mathrm{ETFE}}$ and $m_{\mathrm{DVB}}=\gamma m_{\mathrm{PS}}$, where $\gamma$ is an effective crosslink level, which in general might differ from the level of the initial monomer solution. ${ }^{[16]}$ The degree of sulfonation is also crosslink dependent, though no dramatic variation is found over the investigated range of crosslink levels. ${ }^{[18]}$ If sulfonation (S) is hundred percent, each styrene moiety is sulfonated and $m_{\mathrm{S}}=\alpha m_{\mathrm{PS}}$ where $\alpha=0.762$. We obtain the value of $\alpha$ based on the molar mass of styrene and that of sulfonated styrene. The water uptake is defined as $W=\left(m^{\text {wet }}-m^{\text {dry }}\right) / m^{\text {dry }}$, where $m^{\text {wet }}$ and $m^{\text {dry }}$ are the weight of the membrane before and after swelling. We found earlier that the relationship between water uptake and graft level is quadratic, ${ }^{[35,36]}$ while the dependence on the crosslink level is hyperbolic. ${ }^{[16,18]}$ Using the simple mass model outlined above and applying these findings, we can write an empirical formula quantifying water uptake

$$
W\left(G_{1}, \gamma\right) \simeq \frac{\left[c_{0}+G_{1}\left(c_{1}+c_{2} G_{1}\right)\right](1+\gamma)}{\left(d_{0}+d_{1} \gamma\right)\left[1+\gamma+G_{1}(1+\alpha+\gamma)\right]}
$$

where the values of the constants, $c_{i}, d_{i}$, can be obtained by the best fit of the water uptake data (Figure 10).

In our swollen membranes we found a strong correlation between the proton conductivity and the total volume fraction of water (Figure 11). This correlation is well described by a power law and the best fit yields $t=1.71 \pm 0.05$ and $v_{c}=9 \pm 3 \%$. Even though the morphology of our membranes is anisotropic and the crosslink level varies along the thickness, the result of the best fit is in good agreement with the values found in the literature for isotropic homogeneous systems. ${ }^{[32]}$ The power law suggests a random network of aqueous clusters, and it is also highlighted that the aqueous cluster percolation is not far from threshold yet continuous and connected throughout the whole membrane volume.

While the IEC (defined as the number of $-\mathrm{SO}_{3} \mathrm{H}$ exchange sites per the dry mass of polymer membrane) of our membranes decreases only marginally, the proton conductivity, hydration number ( $\lambda$, defined as the number of water molecules per sulfonic acid site), and water uptake are inversely proportional to the crosslink level (Figure 10). ${ }^{[16]}$ The proton concentrations in the membranes synthesized with various crosslink levels are not expected to be significantly different, ${ }^{[37]}$ which is important, because 

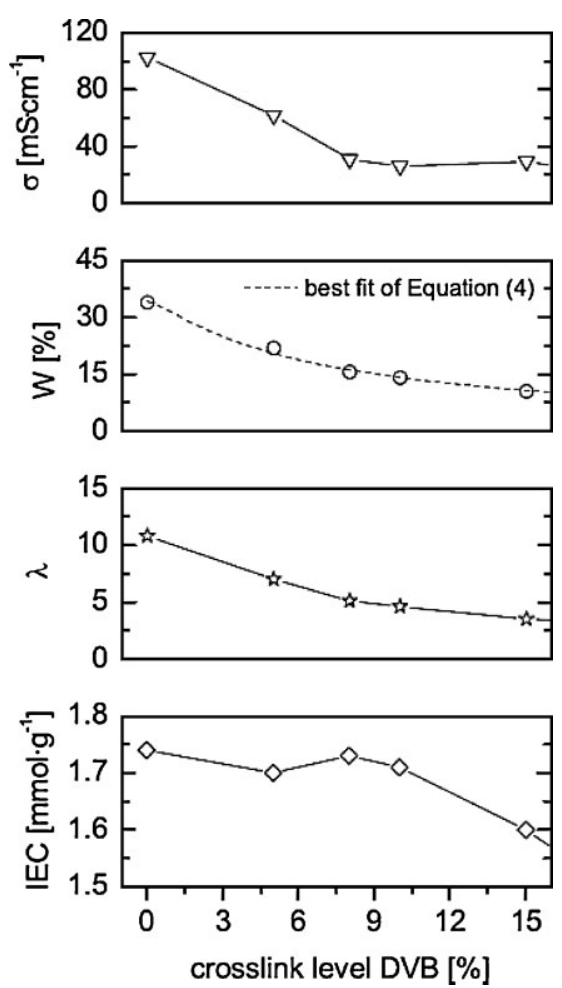

Figure 10. Proton conductivity $\sigma$, water uptake $W$, hydration number $\lambda$, and IEC versus crosslink level at room temperature. ${ }^{[16]}$ The dashed red line is the best fit of Equation (4).

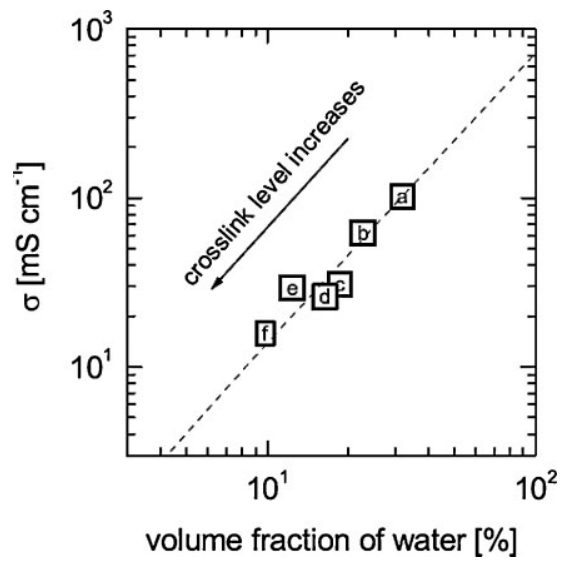

Figure 11. Proton conductivity ${ }^{[16]}$ versus volume fraction of water in the swollen membranes. The dashed line is the best fit of the power function. The crosslink level is (a) o, (b) 5, (c) 8, (d) 10, (e) 15, and $(\mathrm{f}) 20 \%$. The volume fraction of water is calculated from Equation (4).

the Nernst-Einstein equation states that proton conductivity is proportional to the volumetric density of protons and proton diffusion coefficient. ${ }^{[38]}$ In our membranes only the protons are mobile while the counter anions are a part of the immobile matrix. We may define an "effective" proton mobility, which in general is expected to be smaller than the local mobility. For example, a proton found in an aqueous dead-end channel has a good "local" mobility, yet it is not able to travel beyond the channel end. The effective diffusivity of the protons in our membranes exhibits clear dependence on the DVB level, as the introduction of crosslinks directly affects the membrane morphology and the potential level of hydration. The morphology has been shown to play a decisive role for proton conduction in partially sulfonated block copolymer ${ }^{[39]}$ and sulfonated diblock copolymer membranes. ${ }^{[40]}$ The level of hydration obviously has a crucial role, as the proton mobility is governed by the nature of percolation of the aqueous clusters, e.g., pore tortuosity and connectivity. Furthermore, the transport mechanism of protons is also hydration dependent. ${ }^{[3,41,42]}$

\section{Conclusion}

We have investigated the relationship between morphology, hydration, and proton transport in ETFE-based radiation-grafted copolymer proton exchange membranes. We find an anisotropic, dominantly biphasic morphology separating into hydrophilic and hydrophobic domains, mostly following the structure already defined in the semicrystalline base film. The SANS data do not indicate any segregation within the amorphous phase, which one would expect as a result of immiscibility between PS and ETFE. The amorphous phase hosts the water and swells upon hydration. The hydration and the diffusion coefficient of the protons are inversely proportional to the crosslink level. The relationship between proton conductivity and total volumetric fraction of water follows a power law, which indicates a fully percolated and most likely random network of finely dispersed aqueous pores, existing only within the amorphous phase.

Acknowledgements: Sandor Balog gratefully acknowledges the financial support of DANSCATT supported by the Danish Science Council for Natural Sciences. Urs Gasser gratefully acknowledges financial support from the Adolphe Merkle Foundation.

Received: September 18, 2009; Published online: January 7, 2010; DOI: $10.1002 /$ macp.200900503

Keywords: graft copolymers; percolation; polyelectrolytes; proton exchange membranes; small-angle neutron scattering

[1] L. Gubler, G. G. Scherer, Adv. Polym. Sci. 2008, 215, 1.

[2] K. Schmidt-Rohr, Q. Chen, Nat. Mater. 2008, 7, 75.

[3] K. D. Kreuer, J. Membr. Sci. 2001, 185, 29.

[4] US 7396880 (2008), Arkema Inc., invs.: J. Goldbach, S. Gaboury, R. Umpleby, J. Parvole, D. Mountz.

[5] C. K. Shin, G. Maier, B. Andreaus, G. G. Scherer, J. Membr. Sci. 2004, 245, 147 
[6] A. Roy, M. A. Hickner, X. Yu, Y. Li, T. E. Glass, J. E. McGrath J. Polym. Sci., Part B: Polym. Phys. 2006, 44, 2226.

[7] N. Cornet, O. Diat, G. Gebel, F. Jousse, D. Marsacq, R. Mercier M. Pineri, J. New Mater. Electrochem. Syst. 2000, 3, 33.

[8] A. Bhattacharya, B. N. Misra, Prog. Polym. Sci. 2004, 29, 767.

[9] M. M. Nasef, E. S. A. Hegazy, Prog. Polym. Sci. 2004, 29 499.

[10] L. Gubler, S. Alkan Guersel, G. G. Scherer, Fuel Cells 2005, 5, 317.

[11] S. Alkan Guersel, L. Gubler, B. Gupta, G. G. Scherer, Adv. Polym. Sci. 2008, 215, 157

[12] L. Gubler, G. G. Scherer, "Radiation-Grafted Proton Conducting Membranes", in: Handbook of Fuel Cells: Advances in Electrocatalysis, Materials, Diagnostics and Durability, Volume 5 \& 6, H. Yokokawa, W. Vielstich, H. A. Gasteiger, Eds., John Wiley \& Sons, Chichester 2009.

[13] Y. Yang, S. Holdcroft, Fuel Cells 2005, 5, 171.

[14] K. Mortensen, U. Gasser, S. Alkan Guersel, G. G. Scherer J. Polym. Sci., Part B: Polym. Phys. 2008, 46, 1660.

[15] S. Alkan Guersel, H. Ben youcef, A. Wokaun, G. G. Scherer Nucl. Instrum. Methods Phys. Res., Sect. B: Beam Interact. Mater. Atoms 2007, 265, 198.

[16] H. Ben youcef, S. Alkan Guersel, A. Wokaun, G. G. Scherer J. Membr. Sci. 2008, 311, 208.

[17] H. P. Brack, H. G. Buehrer, L. Bonorand, G. G. Scherer, J. Mater. Chem. 2000, 10, 1795.

[18] H. Ben youcef, Radiation Grafted ETFE Based Membranes for Fuel Cells: Improved Mechanical and Oxidative Stability, PhD thesis, Swiss Federal Institute of Technology Zurich 2009, No. 18215.

[19] P. Strunz, K. Mortensen, S. Janssen, Phys. B: Condens. Matter 2004, 350, E783.

[20] J. Chen, M. Asano, Y. Maekawa, M. Yoshida, J. Membr. Sci. 2006, 277, 249.

[21] M. Teubner, R. Strey, J. Chem. Phys. 1987, 87, 3195.

[22] G. Gompper, M. Schick, Phys. Rev. E 1994, 49, 1478.
[23] D. Roux, C. Coulon, M. E. Cates, J. Phys. Chem. 1992, 96, 4174.

[24] G. Porte, J. Phys. Condens. Matter 1992, 4, 8649.

[25] U. Olsson, H. Wennerstrm, Adv. Colloid Interface Sci. 1994, 49, 113.

[26] A. Guinier, G. Fournet, Small-Angle Scattering of X-rays, Wiley, New York 1955.

[27] K. D. Kreuer, S. J. Paddison, E. Spohr, M. Schuster, Chem. Rev. 2004, 104, 4637.

[28] B. Berkowitz, R. Knight, J. Stat. Phys. 1995, 80, 1415

[29] M. Eikerling, A. A. Kornyshev, J. Electroanal. Chem. 2001, 502, 1.

[30] M. Eikerling, A. A. Kornyshev, U. Stimming, J. Phys. Chem. B 1997, 101, 10807.

[31] M. Eikerling, A. A. Kornyshev, A. M. Kuznetsov, J. Ulstrup, S. Walbran, J. Phys. Chem. B 2001, 105, 3646.

[32] W. Y. Hsu, J. R. Barkley, P. Meakin, Macromolecules 1980, 13 , 198.

[33] R. Mezzenga, J. Ruokolainen, G. H. Fredrickson, E. J. Kramer, Macromolecules 2003, 36, 4466.

[34] R. Mezzenga, J. Ruokolainen, G. H. Fredrickson, E. J. Kramer, D. Moses, A. J. Heeger, O. Ikkala, Science 2003, 299, 1872.

[35] B. Gupta, F. N. Buechi, M. Staub, D. Grman, G. G. Scherer, J. Polym. Sci., Part A: Polym. Chem. 1996, 34, 1873.

[36] H. P. Brack, M. Wyler, G. Peter, G. G. Scherer, J. Membr. Sci. 2003, 214, 1.

[37] M. V. Rouilly, E. R. Koetz, O. Haas, G. G. Scherer, A. Chapiró, J. Membr. Sci. 1993, 81, 89.

[38] P. Choi, N. H. Jalani, R. Datta, J. Electrochem. Soc. 2005, 152, E123.

[39] B. Kim, J. Kim, B. Jung, J. Membr. Sci. 2005, 250, 175.

[40] L. Rubatat, C. Li, H. Dietsch, A. Nykanen, J. Ruokolainen, R. Mezzenga, Macromolecules 2008, 41, 8130.

[41] T. A. Zawodzinski, M. Neeman, L. O. Sillerud, S. Gottesfeld, J. Phys. Chem. 1991, 95, 6040

[42] P. Choi, N. H. Jalani, R. Datta, J. Electrochem. Soc. 2005, 152, A1548. 